\title{
Design and Calibration of an Electrostatic \\ Energy Analyzer-Time-of-Flight Mass \\ Spectrometer for Measurement of \\ Laser-Desorbed Ion Kinetic Energies
}

\author{
Gary R. Kinsel \\ Department of Chemistry and Biochemistry, The University of Texas at Arlington, Arlington, Texas, USA \\ David H. Russell \\ Department of Chemistry, Texas A \& M University, College Station, Texas, USA
}

\begin{abstract}
The design of a hybrid electrostatic energy analyzer-time-of-flight mass spectrometer for measurement of ion kinetic energies produced by laser desorption ionization is presented. The need for experimental evaluation of the calibration and performance of the instrument is discussed and a novel laser multiphoton ionization technique, which allows experimental calibration of the energy bandpass of the electrostatic energy analyzer, is described. Laser multiphoton ionization at varying electric field strengths also allows the effects of electric field distortions on energy resolution of the instrument to be probed. Measurement of the translational energies of ions produced by $266-\mathrm{nm}$ laser desorption ionization at $48 \mathrm{~mJ} / \mathrm{cm}^{2}$ of material adsorbed to a stainless steel probe by using this instrument also is presented. Ion translational energies of $+19 \pm 5,+10 \pm 5$, and $+10 \pm 5 \mathrm{eV}$ are found for adsorbed $\mathrm{Na}^{+}$, $\mathrm{K}^{+}$, and $m$-xylene $\mathrm{M}^{+}$, respectively. (/ Am Soc Mass Spectrom 1995, 6, 619-626)
\end{abstract}

$\Lambda$ ccurate measurement of the kinetic energies of ions ejected under various experimental conditions is important to the development and validation of models that describe the mechanism of laser desorption ionization (LDI) of condensed materials. Ion kinetic energies are an essential component of the system energy balance following laser excitation of the bulk material and can be used to implicate various solid state energy redistribution processes. Furthermore, measured ion kinetic energies can be used to determine the duration and position of ion formation and this information can yield insights into the physical and/or chemical processes that occur during desorption and ionization. Improved models for LDI are important to the development of mass spectrometric techniques based on this methodology, especially matrix-assisted laser desorption ionization (MALDI) [1].

Perhaps the simplest method to determine ion $\mathrm{ki}$ netic energies is the analysis of ion signal profiles in a time-of-flight (TOF) spectrum [2]. If the TOF instrumental parameters (e.g., voltages, drift region lengths, etc.) are known, the flight time of an ion of a given mass and initial kinetic energy can be calculated from first principles. In this manner a TOF spectrum for an

Address reprint request to David $H$. Russell, Department of Chemistry, Texas A \& M University, Laboratory for Biological Mass Spectrometry, College Station, TX 77843-3325. ion of a given mass can be converted into an energy spectrum with the distribution and most probable ion energy determined directly from the ion signal. However, accurate definition of the instrumental parameters is nontrivial and can lead to large errors as the total acceleration applied to the ions increases and as the mass of the ions increases. For example, if the drift region flight path of an ion of $m / z 1000$ accelerated initially across $10,000 \mathrm{~V}$ were in reality $500 \mu \mathrm{m}$ less than a measured $1.0000-\mathrm{m}$ drift region, the early arrival time of the ion would suggest an initial ion translational energy of $10 \mathrm{eV}$ in the TOF to ion kinetic energy conversion. Furthermore, to convert the TOF scale to an ion kinetic energy scale, an assumption must be made regarding either the position or time of ion formation. For LDI experiments ions are either assumed to be formed only during the laser pulse or only at the surface of the desorbed material. However, neither assumption has been verified experimentally and an incorrect assumption can lead to misinterpretation of the ion kinetic energies. For example, ions emitted from the surface tens of nanoseconds after the desorbing laser pulse and ions formed in the gas phase with an acceleration energy deficit will be delayed in arrival at the detector relative to promptly formed ions.

Alternatively, the TOF spectrum can be converted to an ion kinetic energy scale by using the measured flight time(s) of a calibrant ion or ions. It is important 
to recognize that for ion kinetic energy calibration via this method to be meaningful the absolute kinetic energies of the calibrant ions must be correctly defined and calibrant ion formation must occur by the same mechanism that leads to analyte ion formation. Ubiquitous low mass cations such as $\mathrm{Na}^{+}$or $\mathrm{K}^{+}$are commonly chosen as calibrant ions for LDI experiments. These ions are assumed to be desorbed promptly from the surface of the probe with near thermal kinetic energies. There is evidence, however, that formation of these cations may occur by both gas-phase and surface processes [3]. Furthermore, as will be shown in this report, these cations may have kinetic energies greater than thermal energies.

An improved method for determining ion kinetic energies involves scanning the voltage applied to a retarding electric field placed in the flight path of the ions [4]. The number of ions transmitted (or the number of ions reflected) as a function of the difference between the accelerating and retarding electric fields is measured and the distribution of ion kinetic energies is obtained from the first derivative of the measured response. Although the retarding field approach does allow the ion kinetic energies to be measured independently of the duration of ion formation, several limitations can restrict the accuracy of the ion kinetic energy distribution determined by using this approach. At retarding field strengths where large ion signals are recorded, scanning the retarding field results in small changes in a large ion signal. If the error associated with the measurement of a large ion current is also large, then structure within the ion kinetic energy distribution will be lost. In addition, if the ions undergo matastable loss of small neutral molecules in the drift region between the acceleration and retarding field regions, the low energy metastable product ions will be difficult to distinguish from intact ions that have energy deficits. This latter problem becomes more pronounced as the ion mass-to-charge ratio and total acceleration increase and/or as the resolution of the TOF spectrum decreases.

The method commonly used in sector mass spectrometers to measure ion kinetic energies involves scanning the voltages applied to the plates of an electrostatic analyzer (ESA) placed in the ion flight path while restricting the transmission of high and low kinetic energy ions through a set of mechanical apertures [5]. Incorporation of an ESA in a TOF mass spectrometer allows kinetic energies of laser desorbed ions to be measured in a similar fashion. The ion kinetic energy distribution is obtained by measuring the number of ions transmitted at incremental voltage settings of the ESA across the range of ion energies. This approach to measuring laser-desorbed ion kinetic energy distributions is described in several early reports [6]; more recently, ESA-TOF mass spectrometers have been used to measure the kinetic energies of ions desorbed under MALDI conditions [7]. As with the retarding field approach, ion kinetic energies mea- sured with an ESA-TOF mass spectrometer are independent of the temporal duration of ion formation. In addition, errors associated with ion metastable decay are minimized by reducing the drift region between the ion source region and the ESA without sacrificing resolution of the detected ion signal. However, the mechanical apertures at the entrance and exit of the ESA lead to a reduction in the total ion current transmitted at a given setting and ultimately to reduced sensitivity for the ESA-TOF. On the other hand, restriction of both the high and low ion translational energies transmitted proves an advantage for examination of the details of the ion translational distribution. This advantage is achieved since the number of ions produced within a given energy range is measured directly rather than determined from the difference between measured ion currents as a function of retarding field voltage. A further challenge associated with the use of an ESA-TOF mass spectrometer for the measurement of ion translational energies is that both the absolute ion energy scale and the energy bandwidth of the instrument must be determined.

In this report we describe the design of a hybrid ESA-TOF mass spectrometer suitable for LDI experiments, the calibration of the absolute ion energy scale, and a novel laser multiphoton ionization method for calibration of the instrument energy bandwidth. The considerable challenges that are encountered in making accurate measures of ion translational energies require a thorough evaluation of instrument performance prior to application of the measurement methodology. In the reported experiments, the contrast between the calculated and experimentally determined values for the energy bandpass of the ESA-TOF mass spectometer and the direct evidence for the strong influence of fringing electric fields underscores the need for experimental evaluation of instrument calibration. Furthermore, application of the ESA-TOF mass spectrometer to the measurement of most probable ion translational energies for $\mathrm{Na}^{+}, \mathrm{K}^{+}$, and $m$-xylene produced by low energy 266-nm LDI of material adsorbed to a stainless steel probe surface leads to measured energies of $+19 \pm 5,+10 \pm 5$, and $+10 \pm 5 \mathrm{eV}$, respectively. These results contradict the assumption of near thermal translational energies for "simple" LDI of alkali ions and emphasize the need for caution when laser-desorbed alkali ions are used to calibrate ion translational energies.

\section{Experimental}

A diagram of the ESA-TOF mass spectrometer developed for these studies is shown in Figure 1. The instrument consists of the source housing, ion source region, and $380-\mathrm{mm}$ radius $90^{\circ}$ ESA taken from an MS-9 mass spectrometer (Kratos Analytical, Ramsey, NJ). Modifications to the source housing include the addition of a high vacuum ball valve to allow an insertion probe to enter the source chamber from di- 


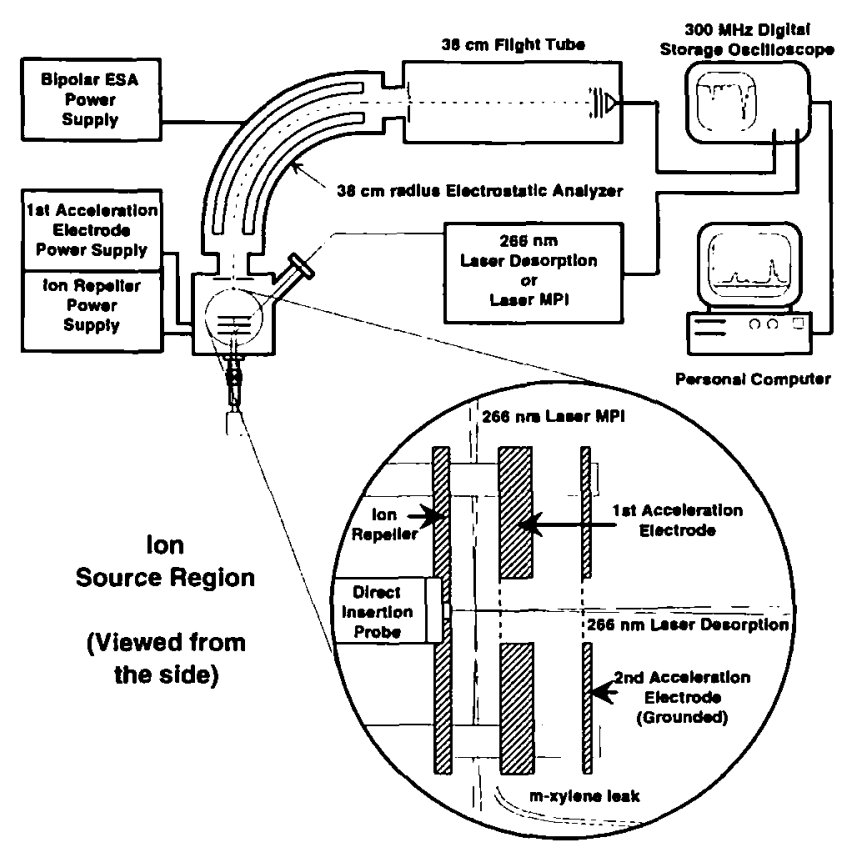

Figure 1. Diagram of the ESA-TOF mass spectrometer developed to measure laser-desorbed ion kinetic energies, 266- $\mathrm{nm}$ laser radiation may be used either for MPI of gas-phase $m$-xylene or for LDI from the stainless steel tip of the direct insertion probe. The inset shows an expanded view (not to scale) of the modified ion source region. See the text for further instrumental details.

rectly behind the ion source region, an angled side arm with an optical port for transmission of the desorbing laser radiation, and two optical ports centered above and below the ion source region to allow gas phase laser ionization to be performed within the ion source region. A $380-\mathrm{mm}$ flight tube has been added to the exit assembly of the ESA to increase to $60 \mathrm{~mm}$ the distance the ions travel after exiting the ESA. A dual microchannel plate detector is used to detect ions transmitted by the ESA.

An expanded view (not to scale) of the modified ion source region is shown in the inset of Figure 1. The ion acceleration assembly consists of three 50-mm-diameter stainless steel electrodes. The tip of the direct insertion probe is inserted through a hole in the center of the ion repeller until the surface of the probe tip is flush with the surface of the repeller electrode. A $90 \%$ transmission nickel mesh (Buckbee-Mears, Munich, Germany) is attached to the side of the first acceleration electrode, facing the ion repeller by using conductive silver paint. A second electrode with a $90 \%$ transmission nickel mesh attached to the side facing the first acceleration electrode defines the exit of the ion acceleration assembly. Each of the three electrodes is separated by four 10-mm nylon spacers. Independently variable voltages may be applied to both the ion repeller and first accelerating electrode to allow either one- or two-stage ion acceleration. The second acceleration electrode is maintained at ground potential for all experiments. No additional focusing lenses or de- flection plates are incorporated in the mass spectrometer source region design. Under two-stage acceleration conditions the combination of the slot milled in the relatively thick first accelerating electrode and the nickel mesh creates an electrostatic ion lens that can partially focus the laser-desorbed ions onto the entrance slit of the ESA dependent on the accelerating voltages applied.

Ions may be generated by either laser ionization of gas phase neutral molecules or by LDI of solid samples applied to the insertion probe. For gas phase ionization experiments a molecular leak valve is used to introduce $m$-xylene into the ion source region to a partial pressure of $\sim 5 \times 10^{-6}$ torr. The frequency-quadrupled output of a pulsed Nd:YAG laser (Quanta-Ray GCR11, Spectra Physics, Mountain View, CA) at 266 $\mathrm{nm}(h \nu=4.66 \mathrm{eV})$ is focused into the center of the first acceleration region of the ion source by using a $150-\mathrm{mm}$ quartz cylindrical lens aligned so that the focused laser beam is parallel to the accelerating electrodes. At typical ionizing laser energies of $\sim 200-\mu \mathrm{J} /$ pulse resonant two photon absorption by the $m$-xylene neutral molecules leads to the formation of positively charged intact and fragment ions [8]. The $m$-xylene positive ions generated in this fashion are accelerated out of the ion source region and travel a distance of $200 \mathrm{~mm}$ before entering the $380-\mathrm{mm}$ radius ESA. An adjustable mechanical entrance slit restricts the collection aperture of the ESA and is maintained at an opening of $\sim \pm 0.23 \mathrm{~mm}(0.46-\mathrm{mm}$ total opening). Turning the ions through $90^{\circ}$ is accomplished by the application of matched positive and negative voltages to a pair of 76-mm wide $90^{\circ}$ arc stainless steel electrodes separated by $12.7 \mathrm{~mm}$. The potentials applied to the ESA electrodes may be adjusted independently of the voltages applied to the ion source region. The energy bandpass of the ESA is controlled through adjustments of the mechanical entrance $(\alpha)$ slits and a second set of mechanical slits positioned at the exit $(\beta)$ of the ESA. Ions transmitted through the ESA traverse the $380-\mathrm{mm}$ field-free drift tube where they are postaccelerated 2.25 $\mathrm{kV}$ before being detected. A photodiode positioned to detect a reflection of the ionizing laser pulse triggers a digital storage oscilloscope (LeCroy 9450, LeCroy, Chestnut Ridge, NY) that monitors the transient output that results from ions that strike the detector. For each ionizing laser pulse an entire mass spectrum is recorded and displayed on the oscilloscope.

Nearly identical operating conditions are used for laser desorption experiments. The 266-nm laser beam is focused via a $500-\mathrm{mm}$ spherical lens through the angled side arm on the source housing onto the surface of the direct insertion probe. The irradiated probe surface appears as a horizontal stripe $\sim 1.0 \times 0.25$ $\mathrm{mm}$. Incident laser intensity is controlled by adjustment of the voltage applied to the flashlamps to give typical desorbing laser energies of $\sim 120 \mu \mathrm{J} /$ pulse $\left(48 \mathrm{~mJ} / \mathrm{cm}^{2}\right)$. The positive ions generated by the pulsed laser radiation are accelerated across one or two stages 
of acceleration, enter the ESA where ion kinetic energy selection is accomplished, and are then detected in a manner identical to that described for the gas phase ionization experiments.

\section{Instrument Calibration}

Calibration of the ESA-TOF mass spectrometer encompasses two important issues-absolute assignment of the ion translational energies relative to the total acceleration potential and determination of the distribution of ion translational energies transmitted at a given set of instrumental conditions. For a singly charged ion, the radius of curvature of the ion trajectory in a field applied perpendicular to the ion motion is given by $r=2\left(A \pm E_{k}\right) / F_{\perp}$, where $A$ is the ion acceleration energy, $E_{k}$ is the initial ion translational energy, and $F_{\perp}$ is the field applied perpendicular to the direction of ion motion. Thus absolute assignment of the ion translational energies is dependent on a knowledge of the radius of the ESA and the accuracy of the measurement of the high voltages applied to the ion source region and to the ESA electrodes. Considerable effort was made to assess the uncertainty in the measurement of the voltages applied to the source region and ESA electrodes measured by using a voltmeter (Fluke 77) and high voltage probe (Keithley 1600, Keithley, Cleveland, $\mathrm{OH}$ ). Initially, the accuracy of the voltmeter was confirmed at low voltages $(1-10 \mathrm{~V})$ by using a calibrated voltage standard (Simpson 1700, Simpson Electric Co., Chicago, IL). Next a resistor string of $\sim 70 \mathrm{M} \Omega$ was constructed from numerous $1 \%$ resistors whose resistance's were individually measured by using a calibrated $5 \frac{1}{2}$ digit Fluke $8840 \mathrm{~A}$ multimeter. A voltage comparable to the accelerating voltage of the ESA-TOF mass spectrometer was applied to the resistor string and the total voltage applied was measured by using the Fluke 77 voltmeter and Keithley high voltage probe. Simultaneously, the voltage drop across a resistor in the voltage divider that represented $1 / 3532.46$ was measured directly by using a second calibrated voltmeter, and the total voltage applied to the resistor chain was calculated. The calculated voltage applied was compared to the voltage measured via the high voltage probe and found to agree to better than $\pm 5 \mathrm{~V}$. This evaluation was repeated by using several different total resistance loads and small fraction voltage dividers to confirm the limits of the error in the high voltage measurement. Propagation of the error through all voltage measurements leads to a total error of $\pm 5 \mathrm{~V}$ in the absolute ion translational energy transmitted at a given set of ESA-TOF mass spectrometer potentials. Additional confirmation of the accuracy of the measured ESA-TOF mass spectrometer potentials and the resultant assignment of ion translational energies relative to the accelerating potential was obtained from independent flight time analyses of MALDI ion signals [9].

The distribution of ion translational energies trans- mitted at a given set of ESA-TOF mass spectrometer instrumental conditions is significantly more difficult to determine. For the ideal case of a collimated beam of ions that originate from a point source, the distribution of transmitted ion translational energies is determined simply by the width of the opening of the $\beta$ slit aperture positioned at the exit of the ESA. The ion translational energy distribution may be calculated from the instrument geometry and aperture size (geometric energy bandpass). However, for most instruments and ionization techniques the ideal case of a collimated beam of ions that originate from a point source is not realized. Penetrating fields at grids or around ion electrodes can distort ion beam collimation [7a]. Furthermore, for LDI experiments ions may be desorbed with large distributions of both forward directed and radial velocities [10]. A large spread in ion angular trajectories that enter the ESA, whether as a result of electric field distortions or the specific mechanism of ion formation, results in loss of energy definition by the $\beta$ slit. For example, ions with translational energies greater than the geometric energy bandpass may still be transmitted if the ions enter at an angle that penetrates into the repulsive ESA potential. This problem can be partially corrected by restricting the ion angular trajectory distribution collected through the use of the $\alpha$ slit at the entrance of the ESA, and this approach must be considered essential if high resolution ion energy measurements are to be made. Ultimately, however, attenuation of the ion current places a lower limit on the opening of the $\alpha$ slit.

The uncertainties associated with the geometric definition of ESA energy bandpass stimulated us to explore a novel experimental approach to determining this value by laser multiphoton ionization (MPI) of gas-phase molecules. For a given set of ion formation conditions, the ion kinetic energy distribution observed, $\left\langle E_{\text {obs }}\right\rangle$, by scanning the potential applied to the ESA will comprise the sum of the energy bandpass of the ESA including any distortions introduced by the ionization or collection of ions, $\left\langle E_{\text {esa }}\right\rangle$, plus the initial energy distribution of the ions, $\left\langle E_{\text {ion }}\right\rangle$.

$$
\left\langle E_{\text {obs }}\right\rangle=\left\langle E_{\text {esa }}\right\rangle+\left\langle E_{\text {ion }}\right\rangle
$$

$\left\langle E_{\text {ion }}\right\rangle$ will comprise the sum of the initial translational energies of the ions $\left\langle E_{\mathrm{T}}\right\rangle$ plus the product of the source region field strength $\left(F_{\mathrm{s}}\right)$ and the spatial width over which the ions are formed (dS). At room temperature the one-dimensional translational energies of gas-phase molecules are small $\left(\frac{1}{2} R T \cong 0.01 \mathrm{eV}\right)$; thus ions formed from these molecules will have similar small $\left\langle E_{\mathrm{T}}\right\rangle$. The exception to this relation is if ion production is accompanied by substantial kinetic energy release. This latter complication is avoided by analysis of radical cations produced by laser MPI since large kinetic energy release is not associated with ejection of an electron from a neutral molecule [11]. On the other hand, the ion energy distribution associated with the product of $F_{\mathrm{s}}$ and $d S$ can be large (tens to hun- 
dreds of volts) given the finite width of the laser focal point $(>100 \mu \mathrm{m}$ ) and the high source region extraction field strengths (hundreds to thousands of volts per centimeter). Thus, for $\left\langle\mathrm{E}_{\text {ion }}\right\rangle \cong F_{\mathrm{s}} * d S$, substitution into eq 1 gives

$$
\left\langle E_{\mathrm{obs}}\right\rangle=\left\langle E_{\mathrm{esa}}\right\rangle+F_{\mathrm{s}} * d S
$$

From eq 2 it can be seen that for ion energy distributions measured across a series of source region extraction field strengths, the resultant $\left\langle E_{\text {obs }}\right\rangle$ plotted versus Fs should yield a straight line with an intercept of $\left\langle E_{\text {esa }}\right\rangle$ and a slope of $d S$. In this manner a direct experimental measure of the ESA energy bandpass can be obtained.

To calibrate the modified ESA-TOF mass spectrometer instrument we performed 266-nm MPI of gasphase room temperature $m$-xylene admitted to the ion source region via a molecular leak valve. The voltage applied to the ion repeller was held constant at \pm 7940 $\mathrm{V}$ (all high voltages are measured to $\pm 5 \mathrm{~V}$ ). The voltage applied to the first acceleration plate was varied from $+6940 \mathrm{~V}$ to $+7840 \mathrm{~V}$ in increments of $100 \mathrm{~V}$ and the second acceleration plate was held at ground potential. At each first acceleration plate potential the ion energy distribution, $\left\langle E_{\mathrm{obs}}\right\rangle$, was measured by scanning the potentials applied to the ESA. Figure 2 shows a series of segments of the TOF spectra for the $\mathrm{M}^{+\cdot}$ of $m$-xylene at a source region $F_{\mathrm{s}}=1000 \mathrm{~V} / \mathrm{cm}$ taken at $0.2-\mathrm{V}$ incremental settings of the ESA potential. Each spectrum along the $z$ axis corresponds to a $3-\mathrm{eV}$ change in ion energy transmitted by the ESA. $\left\langle E_{\text {obs }}\right\rangle$ is obtained from the difference between the maximum and minimum ion energy transmitted by the ESA. Figure 3 shows a plot of $\left\langle E_{\text {obs }}\right\rangle$ versus $F_{s}$ with the ESA $\alpha$ slit nominally at $\pm 0.23 \mathrm{~mm}$ and $\beta$ slit nominally at \pm 0.25 $\mathrm{mm}$. The error bars represent the average deviation of

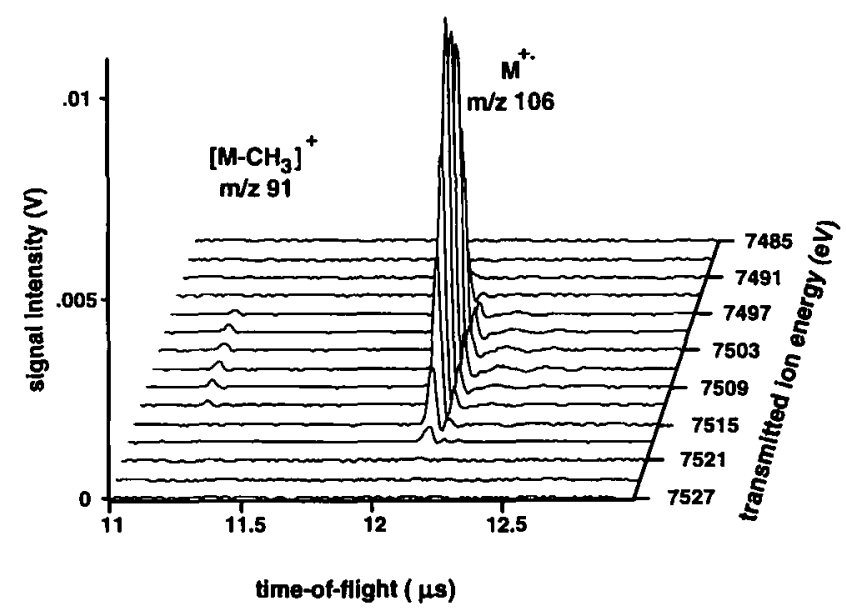

Figure 2. Partial TOF spectra of $m$-xylene ions produced by 266-nm MPI of room temperature molecules recorded at incremental settings of the ESA potential. The $z$ axis represents the total kinetic energy of the ions as calculated from the potential applied to the ESA. See the text for instrumental conditions used for spectra acquisition.

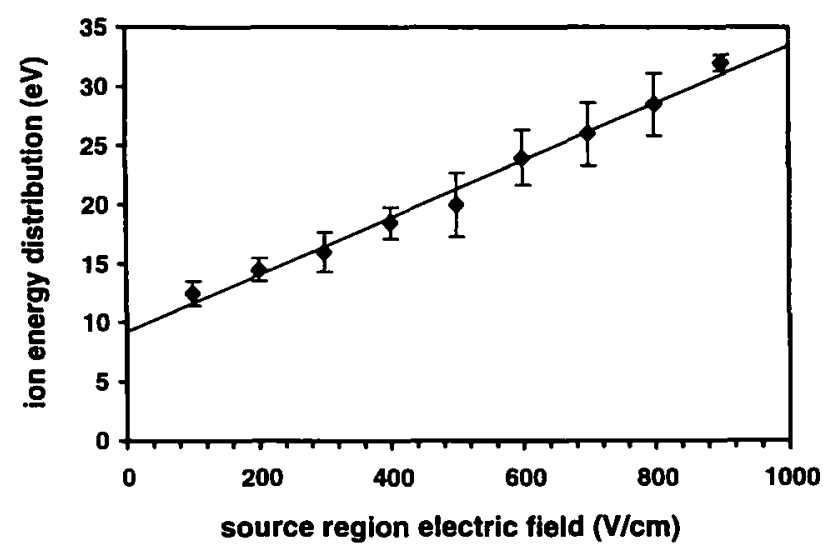

Figure 3. Ion kinetic energy distributions for $m$-xylene measured as a function of source region electric field strength. The $m$-xylene ions were produced by 266-nm MPI. The $y$ intercept of the plot gives a bandpass energy of $9.2 \mathrm{eV}$ for the ESA.

three separate runs. The intercept of this plot gives $\left\langle E_{\text {esa }}\right\rangle=9.2 \mathrm{eV}$ and the slope gives $d S=0.24 \mathrm{~mm}$. The $\left\langle E_{\text {esa }}\right\rangle$ value of $9.2 \mathrm{eV}$ determined from the experimental measurements with $\beta= \pm 0.25 \mathrm{~mm}$ is in strong contrast to the calculated geometric energy bandpass of $\left\langle E_{\text {esa }}\right\rangle=5.3 \mathrm{eV}$.

Calibration studies performed at several settings of the $\beta$ slit aperture and laser focal widths yield results consistent with expectations. Increases in the $\beta$ slit aperture gave incremental increases in $\left\langle E_{\text {esa }}\right\rangle$ and increases in the laser focal width yielded calibration plots with steeper slopes. Changes in the total ion acceleration potential at fixed $\alpha$ and $\beta$ slit apertures also resulted in changes in the measured $\left\langle E_{\text {esa }}\right\rangle$ as expected. For example, reduction of the total ion acceleration from 8 to $6 \mathrm{kV}$ at $\alpha$ and $\beta$ apertures identical to those used in Figure 2 yielded a change in measured $\left\langle E_{\text {esa }}\right\rangle$ from 9.2 to $5.9 \mathrm{eV}$. Note that even at $6-\mathrm{kV}$ acceleration the measured $\left\langle E_{\text {esa }}\right\rangle$ of $5.9 \mathrm{eV}$ remains larger than the calculated geometric energy bandpass of $4.0 \mathrm{eV}$. The discrepancy between the experimental and calculated energy bandpass of the ESA most likely results from poor ion beam collimation, but cannot be rationalized in terms of a large distribution of initial ion angular trajectories. As discussed earlier, radical cations produced by MPI of room temperature gasphase molecules have very low translational energies. Although the volume in which ions are formed may be quite large due to ionization in the focused laser beam waist, the $200-\mathrm{mm}$ distance between the point of MPI and the low radial translational energies should yield an almost ideal collimated beam illumination of the $\alpha$ slit of the ESA.

Further insight into the energy bandpass discrepancy was obtained from an examination of the ion energy distributions measured at low source region extraction field strengths. As the source region field strength is reduced below $200 \mathrm{~V} / \mathrm{cm}$ the ion energy distributions remain consistent with values expected from extrapolations of the calibration plots obtained at 
higher source region field strengths. However, the point of ionization, as calculated from the most probable ion energies, the voltage drop across the source region, and the measured source region width, appears to change. Figure 4 shows the position of ion formation calculated from measured most probable ion energies for source region field strengths that ranged from 20 to $200 \mathrm{~V} / \mathrm{cm}$ with the ion repeller set at $+7940 \mathrm{~V}$ and a nominal source region width of $1 \mathrm{~cm}$. It appears from these calculations that the point of ionization moves toward the ion repeller as the field strength decreases. Because the physical position of the laser beam focus is not being adjusted, this result must reflect the influence of penetrating electric fields from the second acceleration region at very low source region field strengths. Similar electric field distortions must be expected in the secondary acceleration region under reverse conditions, that is, when the field is large in the source region and small in the secondary acceleration region. This result suggests that the discrepancy between the experimental and calculated ESA energy bandpass is a result of loss of ion beam collimation due to electric field distortions in and around the source region accelerating electrodes.

\section{Desorption Ion Kinetic Energies}

After calibration of the ESA-TOF mass spectrometer as described above, the energy distribution for ions produced by $266-\mathrm{nm}$ irradiation of alkali metals and $\mathrm{m}^{-}$ xylene adsorbed on a stainless steel probe tip were measured to test the performance of the instrument. The $\alpha$ and $\beta$ slits of the ESA were set at $\pm 0.23 \mathrm{~mm}$ and $\pm 0.25 \mathrm{~mm}$ respectively-identical to the laser MPI experiments. Because the ions produced by 266$\mathrm{nm}$ LDI travel a nearly identical distance prior to

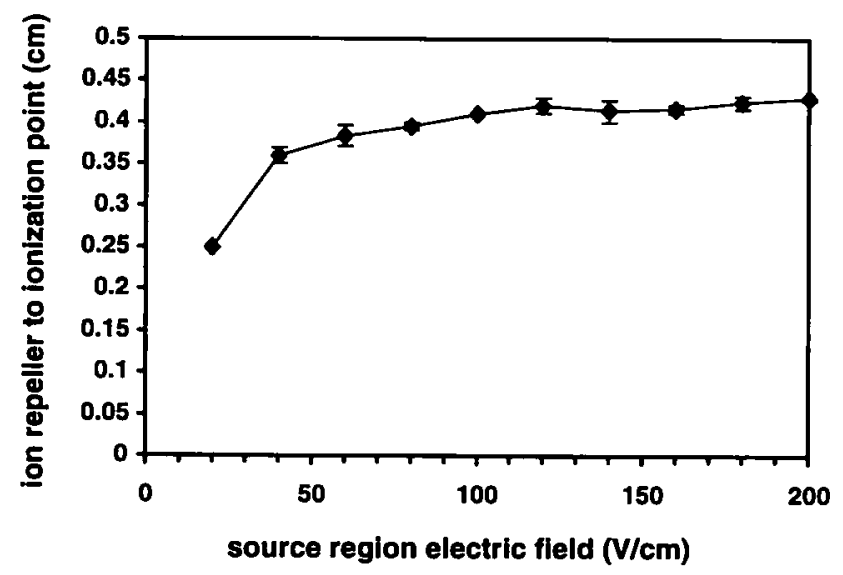

Figure 4. Calculated distance from the ion repeller to the point of ionization (266-nm laser focal point) as a function of source region electric field strength. The distance is calculated from the measured most probable ion kinetic energy, the width of the source region, and the voltages applied to the ion repeller and first acceleration electrode. The apparent movement of the point of ionization is a result of electric field distortions at low source region electric fields. entering the ESA and because the LDI ions must experience similar electric field distortions in the source region, the energy bandpass of the ESA is likely identical to the $9.2 \mathrm{eV}$ determined for these $\alpha$ and $\beta$ settings by the MPI experiments. The absolute ion translational energies relative to the acceleration potential were measured to $\pm 5 \mathrm{~V}$. The ion repeller voltage was reduced slightly and held constant at $+7775 \mathrm{~V}$ to give the surface-desorbed ions a similar total acceleration as the ions formed in the gas-phase MPI calibration studies. The first and second acceleration grids were held at ground potential. Figure 5 shows a series of segments of the 266-nm LDI-TOF spectra taken at 0.6-V incremental settings of the ESA potential. Each spectrum along the $z$ axis corresponds to a 9.0-eV change in ion energy transmitted by the ESA, which is nearly equivalent to the energy bandpass of the instrument. Figure 5a shows ion signals for $\mathrm{Na}^{+}$and $\mathrm{K}^{+}$that originate from the stainless steel probe. Figure $5 b$ shows the ion signals for $m / z 106\left(\mathrm{M}^{+\cdot}\right)$ and $m / z 91$ $\left(\left[\mathrm{M}-\mathrm{CH}_{3}\right]^{+}\right)$for $m$-xylene. These ion signals represent the major signals observed in the LDI-TOF mass
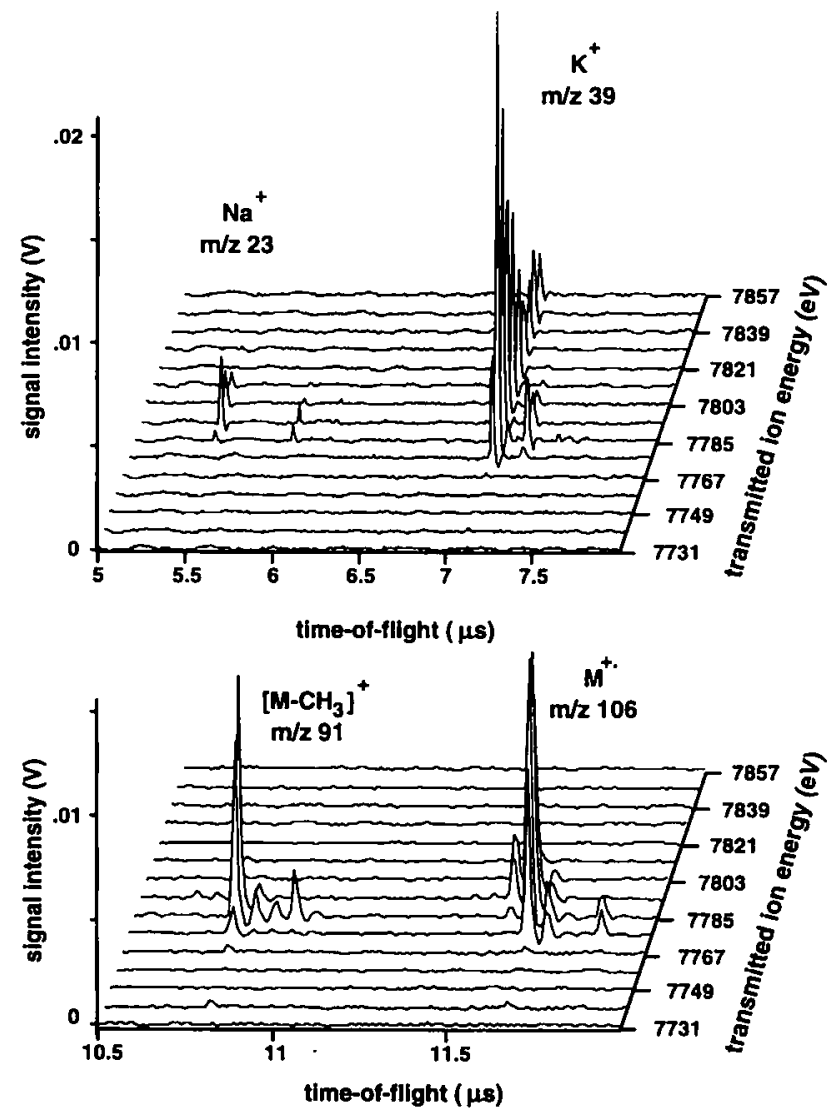

Figure 5. Partial TOF spectra of ions produced by 266-nm LDI from a stainless steel probe tip recorded at incremental settings of the ESA potential. The ion repeller voltage was held constant at $+7775 \mathrm{~V}$. The $z$-axis represents the total kinetic energy of the ions as calculated from the potential applied to the ESA. Regions of the TOF spectra contain (a) $\mathrm{Na}^{+}$and $\mathrm{K}^{+}$and (b) ions from adsorbed $m$-xylene at $m / z 106$ and 91 . See the text for instrumental conditions used to spectra acquisition. 
spectra; other regions of the TOF mass spectra have been omitted for clarity.

To examine the ion energy distributions more closely, the ion signal intensities recorded at each ESA setting are integrated and the normalized values are plotted versus the absolute ion energy relative to the acceleration potential (Figure 6). The plotted values do not represent ion signal intensities measured at a fixed flight time because the flight times of the ion signals shift as a function of the ion energy. For each plot the displayed points represent the average integrated signal intensity for two separate runs. For $\mathrm{Na}^{+}$the most probable ion energy is $+19 \pm 5 \mathrm{eV}$ and for $\mathrm{K}^{+}$the most probable ion energy is $+10 \pm 5 \mathrm{eV}$ with an extended high energy tail (Figure 6a). We have been unable to find literature citations of identical UV LDI ion kinetic energy measurements for $\mathrm{Na}^{+}$and/or $\mathrm{K}^{+}$ with which to compare our results. An apparent discrepancy exists between our measured value of $+19 \pm$ $5 \mathrm{eV}$ for 266-nm LDI of $\mathrm{Na}^{+}$and previously reported values of $\equiv 1 \mathrm{eV}$ for IR LDI of $\mathrm{Na}^{+}$[12]. However, numerous reports of similar visible and UV wavelengths for LDI have noted high kinetic energies for small metal cations. In early work by Hillenkamp et al.
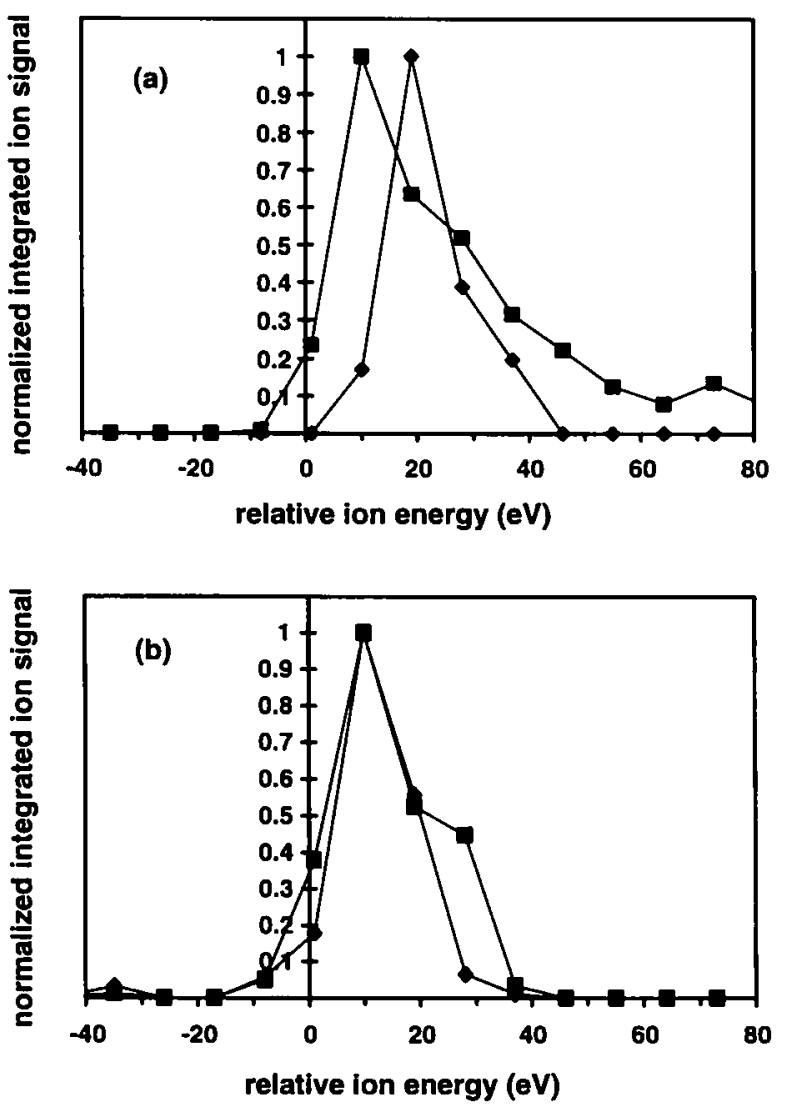

Figure 6. Normalized integrated in signals produced by $266-\mathrm{nm}$ LDI from a stainless steel probe tip recorded as a function of the ion energy relative to the applied source region repeller voltage. Ion energy distributions measured for $(a) \mathrm{Na}^{+\cdot}(\bullet)$ and $\mathrm{K}^{+\cdot}(\boldsymbol{\square})$ and for (b) $m / z \quad 106(\mathbb{a})$ and $m / z 91(\diamond)$ produced from adsorbed $m$-xylene.
[13] most probable ion energies at $\lambda=347 \mathrm{~nm}$ of \pm 5 $\mathrm{eV}$ for $\mathrm{Na}^{+}$and $+14 \mathrm{eV}$ for $\mathrm{Al}^{+}$. were determined via quadrupole and TOF methods. Subsequently, Hardin and Vestal [14] also reported most probable ion energies $(\lambda=483 \mathrm{~nm})$ of $+6 \mathrm{eV}$ for $\mathrm{K}^{+}$with high energy tails that extend beyond $+25 \mathrm{eV}$. These studies were performed by using TOF and quadrupole energy determination. Michiels et al. [15] reported most probable ion energies $(\lambda=266 \mathrm{~nm})$ of $+10 \mathrm{eV}$ for ${ }^{46} \mathrm{Ti}^{+\cdot}$ by using a retarding field measurement, and these authors report that ion energies for $\mathrm{Na}^{+}, \mathrm{Mg}^{+}, \mathrm{K}^{+}$, and $\mathrm{Ca}^{+\cdot}$ are very similar to that of titanium. Helvajian and Welle [16] also recently have reported a TOF method for energy determination that yielded most probable ion energies $(\lambda=248$ and $351 \mathrm{~nm})$ of $+9 \mathrm{eV}$ for $\mathrm{Ag}^{+}$, $\mathrm{Ag}_{2}^{+}$, and adsorbed $\mathrm{Fe}^{+}$. Our results are generally consistent with these latter measurements within experimental error and may represent an effect of visible or UV versus IR desorption wavelength on desorbed ion translational energies.

For both the $m$-xylene $\mathrm{M}^{+\cdot}$ and $\left[\mathrm{M}-\mathrm{CH}_{3}\right]^{+}$the most probable ion energy is $+10 \pm 5 \mathrm{eV}$. These values for most probable ion energies are consistent with the ion energies measured for $\mathrm{Na}^{+}$and $\mathrm{K}^{+}$. These values are somewhat higher than the value of $+1 \mathrm{eV}$ reported by Yang and Reilly [17] for 260-nm laser-desorbed aniline from gold and aluminum surfaces as determined from TOF measurements. In the same study, however, the authors point out the influence of extraction field strength on ion kinetic energies, and it should be noted that the extraction field strengths (17-175 $\mathrm{V} / \mathrm{cm}$ ) used in their study were much lower than the $7775 \mathrm{~V} / \mathrm{cm}$ used in this study. Studies performed in this laboratory (Kinsel, G. R.; Russell, D. H., manuscript in preparation) also suggest an influence of extraction field strength on measured ion translational energies, that is, measured ion translational energies decrease as extraction field strength is reduced. Further studies of both wavelength and extraction electric field strength effects are needed to gain insight into the mechanism(s) of LDI.

\section{Conclusion}

The ESA-TOF mass spectrometer offers potential advantages for the measurement of ion translational energies over both TOF profile analysis and retarding field approaches. However, calibration of the absolute ion translational energies and energy bandpass of the ESA-TOF mass spectrometer must be performed to obtain meaningful results. In this article we address the issues of absolute ion energy calibration and describe a method to experimentally determine the energy bandpass of the ESA-TOF mass spectrometer by variable two-stage acceleration of ions produced by laser MPI of gas-phase molecules. A discrepancy between the experimentally determined ESA energy bandpass and the energy bandpass calculated based on 
the geometry of the instrument is attributed to poor collimation of the collected ion angular trajectories primarily as a result of electric field distortions. Distortion of accelerating electric fields has been confirmed by the MPI experiments at low source region field strengths through calculation of the position of ion formation from measured most probable ion translational energies. Additional studies of the translational energies of ions produced by 266-nm LDI of adsorbed species on a stainless steel probe also have been performed by using the calibrated ESA-TOF mass spectrometer. Most probable energies for $\mathrm{Na}^{+}$and $\mathrm{K}^{+}$of $+19 \pm 5$ and $+10 \pm 5 \mathrm{eV}$, respectively have been measured with $\mathrm{K}^{+}$that has a high energy tail that extends beyond $+80 \mathrm{eV}$. LDI of adsorbed $m$-xylene at $266 \mathrm{~nm}$ also shows most probable ion energies of $+10 \pm 5 \mathrm{eV}$ for $\mathrm{M}^{+\cdot}$ and $[\mathrm{M}-\mathrm{CH} 3]^{+}$. The high translational energies of the alkali ions produced by $266-\mathrm{nm}$ LDI are in contrast to previous reports of near thermal energies for IR LDI of these species, and this result suggests that caution should be exercised when these ions are used for calibration of ion translational energies. These studies have allowed us to experimentally evaluate the calibration and performance of the ESATOF mass spectrometer and to pave the way for future studies of the translational energies of ions produced by MALDI.

\section{Acknowledgments}

This work was supported by grants from the United States Department of Energy, Division of Chemical Sciences, Office of Basic Energy Science, and the National Science Foundation. We would like to thank M. L. Krumnow for his assistance in analysis of the instrumental parameters.

\section{References}

1. (a) Hillenkamp, F.; Karas, M.; Beavis, R. C.; Chait, B. T. Anal. Chem. 1991, 63, 1193A; (b) Chait, B. T.; Kent, S. B. H. Science 1992, 257, 1885; (c) Karas, M.; Bahr, U.; Giessman, U. Mass Spectrom. Rev. 1991, 10, 335.

2. (a) Tsong, T. T. Int. J. Mass Spectrom. Ion Processes 1986, 70, 1; (b) Ens, W.; Mao, Y.; Mayer, F.; Standing, K. G. Rapid Commun. Mass Spectrom. 1991, 5, 117; (c) Makarov, A. A.; Davis,
S. C.; Greathead, R. J. Proceedings of the 41st ASMS Conference on Mass Spectrometry and Allied Topics; 1993, p 649; (d) Ioanoviciu, D.; Cuna, C.; Ardelean, P. Rapid Commun. Mass Spectrom. 1993, 7, 999.

3. (a) Chiarelli, M. P.; Gross, M. L. Int. I. Mass Spectrom. Ion Processes 1987, 78, 37; (b) Wang, B. H.; Dreisewerd, K.; Bahr, U.; Karas, M.; Hillenkamp, F. J. Am. Soc. Mass Spectrom. 1993, 4, 393; (c) Hogan, J. D.; Laude, D. A. Anal. Chem. 1991, 63, 2105.

4. (a) Mauney, T.; Adams, F. Int. J. Mass Spectrom. Ion Processes 1984, 59, 103; (b) Zhou, J.; Ens, W.; Standing, K. G.; Verentchikov, A. Rapid Commun. Mass Spectrom. 1992, 6, 671.

5. Cooks, R. G.; Beynon, J. H.; Caprioli, R. M.; Lester, G. R. Metastable lons; Elsevier Scientific Publishing: Amsterdam, 1973; pp 37-88.

6. (a) Fenner, N. C.; Daly, N. R. Rev. Sci. Instrum. 1966, 37, 1068; (b) van der Peyl, G. J. Q.; Haverkamp, J.; Kistemaker, P. G. Int. J. Mass Spectrom. Ion Phys. 1982, 42, 125; (c) Pinkston, J. D.; Rabb, M.; Watson, J. T.; Allison, I. Rev. Sci. Instrum. 1986, 57, 583.

7. (a) Giannakopulos, A. E.; Reynolds, D. J.; Chan, T. W. D.; Colburn, A. W.; Derrick, P. J. Int. J. Mass Spectrom. Ion Processes 1994, 131, 67; (b) Brown, R. S.; Bogart, K. H. A.; Dick, D. L. Proceedings of the 41st ASMS Conference on Mass Spectrometry and Allied Topics; 1993, p 949.

8. (a) Lubman, D. Mass Spectrom. Rev. 1988, 7, 535; (b) Lubman, D. Mass Spectrom. Rev. 1988, 7, 559.

9. Kinsel, G. R.; Gillig, K.; Edmondson, R. E.; Russell, D. H. Proceedings of the 42nd ASMS Conference on Mass Spectrometry and Allied Topics; 1994; $\mathrm{p} 4$.

10. Spengler, B.; Boekelmann, V. Nucl. Instrum. Meth. Phys. Res. 1993, 82, 379.

11. Tai, T. L.; El-Sayad, M. A. J. Phys. Chem. 1986, 90, 4477.

12. (a) van der Peyl, G. J. Q.; van der Zande, W. J.; Bederski, K.; Boerboom, A. J. H.; Kistemaker, P. G. Int. I. Mass Spectrom. Ion Phys. 1983, 47, 7; (b) van der Peyl, G. J. Q.; van der Zande, W. J.; Kistemaker, P. G. Int. J. Mass Spectrom. Ion Processes 1984, 62, 51.

13. (a) Hillenkamp, F.; Kaufmann, R.; Nitsche, R.; Remy, E.; Unsoeld, E. Microprobe Analysis as Applied to Cells and Tissues; Hall, T. A.; Echlin, P.; Kaufmann R., Eds.; Academic Press: London, 1974; p 1; (b) Hillenkamp, F.; Unsoeld, E.; Kaufmann, R.; Nitsche, R. Appl Phys. 1975, 8, 341.

14. Hardin, E. D.; Vestal, M. L. Anal. Chem. 1981, 53, 1492.

15. Michiels, E.; Mauney, T.; Adams, F.; Gijbels, R. Int. J. Mass Spectrom. Ion Processes 1984, 61, 231.

16. Helvajian, H.; Welle, R. J. Chem. Phys. 1989, 91, 2616.

17. Yang, M.; Reilly, J. P. J. Phys. Chem. 1990, 94, 6299. 